\title{
Validación de criterio de una herramienta de bajo costo para la detección de la pérdida auditiva infantil en Colombia
}

\author{
Criterion validation of a low-cost tool for detecting childhood hearing loss in \\ Colombia
}

\author{
Validação de critério de uma ferramenta de baixo custo para a detecção da perda \\ auditiva infantil na Colômbia
}

\begin{abstract}
Augusto Muñoz-Caicedo 1 , María Consuelo Chaves-Peñaranda², Claudia Ximena Campo-Cañar ${ }^{3}$.
1 Fonoaudiólogo, magíster en Salud Publica, Universidad del Cauca, Colombia. amunozc@unicauca.edu.co. oRcid: https://orcid. org/0000-0002-0570-5558

2 Fonoaudióloga, audióloga, Universidad del Cauca. Hospital San José, Colombia. mchaves@unicauca.edu.co. ORCID: https://orcid. org/0000-0003-4020-0341

3 Fonoaudióloga, audióloga, Universidad del Cauca, Colombia. xcampo@unicauca.edu.co. ORCID: https://orcid.org/0000-00015352-3065
\end{abstract}

Recibido: 28/03/2019. Aprobado: 08/04/2020. Publicado: 03/07/2020

Muñoz-Caicedo A, Chaves-Peñaranda MC, Campo-Cañar CX. Validación de criterio de una herramienta de bajo costo para detección de la pérdida auditiva infantil en Colombia. Rev. Fac. Nac. Salud Pública. 2020;38(3):e338085. DoI: https:/doi.org/10.17533/ udea.rfnsp.e338085

\section{Resumen}

Objetivo: Establecer la validez de criterio de una herramienta para la detección de la pérdida auditiva en población infantil colombiana. Metodología: Estudio de concordancia en 160 niños entre 5 y 10 años, a quienes se les valoró la audición mediante dos pruebas: la "Herramienta de detección de bajo costo para identificar y clasificar la pérdida auditiva", diseñada en Brasil, y la "Audiometría tonal" como prueba de oro. Se determinó la sensibilidad y la especificidad de la herramienta y la estimación de la concordancia entre las dos pruebas mediante el índice de kappa. Resultados: La "Herramienta de detección de bajo costo para identificar y clasificar la pérdida auditiva" presentó una sensibilidad del $35 \%$, especificidad del $25 \%$, un valor predictivo positivo del $12 \%$, valor predictivo negativo del 56 \% y un índice de kappa de -0,24. Conclusión: La "Herramienta de detección de bajo costo para identificar y clasificar la pérdida auditiva" presenta poca capacidad para clasificar correctamente al enfermo como verdadero enfermo y al sano como verdadero sano. Además, la pobre concordancia entre las dos pruebas no permite clasificarla como una herramienta válida para la identificación y la clasificación de la pérdida 
auditiva en población infantil colombiana. Por lo anterior, se recomienda que cualquier método de tamizaje o de diagnóstico debe cumplir con el proceso de validación antes de ser aplicado en la población. La prevalencia de hipoacusia encontrada fue del $23,1 \%$
--------Palabras clave: Pérdida auditiva, tamizaje masivo, audiometría, validez de las pruebas, reproducibilidad de resultados.

\section{Abstract}

Objective: To establish the criteria validity of a tool to detect hearing loss in Colombian children. Methodology: Concordance study in 160 children between 5-10 years old who had their hearing assessed using two tests: the "Low-cost detection tool to identify and classify hearing loss", designed in Brazil, and the "Tonal Audiometry" as the gold standard. The sensitivity and specificity of the tool and the estimation of the agreement between the two tests were determined using the kappa index. Results: The "Low cost detection tool to identify and classify hearing loss" presented a sensitivity of $35 \%$, specificity of $25 \%$, a positive predictive value of $12 \%$, negative predictive value of $56 \%$ and a kappa index of -0.24 . Con- clusion: The "Low-cost detection tool to identify and classify hearing loss" has little capacity to correctly classify the patient as truly ill and the healthy as truly healthy. Furthermore, the poor concordance between the two tests does not allow it to be classified as a valid tool for identifying and classifying hearing loss in Colombian children. It is therefore recommended that any screening or diagnostic method must comply with the validation process before being applied to the population. The prevalence of hearing loss found was $23.1 \%$

--------Key words: Hearing loss, mass screening, audiometry, test validity, reproducibility of results

\section{Resumo}

Objetivo: Estabelecer a validade de critério de uma ferramenta para a detecção da perda auditiva na população infantil colombiana. Metodologia: Estudo de concordância em 160 crianças com idades entre 5 e 10 anos, cuja audição foi avaliada através de dois testes: a "Ferramenta de detecção de baixo custo para identificar e classificar a perda auditiva" desenhada no Brasil, e a "Audiometria tonal" como exame padrão-ouro. Determinou-se a sensibilidade e a especificidade da ferramenta, e a estimativa entre as duas avaliações foi feita através do coeficiente de concordância Kappa. Resultados: A "ferramenta de detecção de baixo custo para identificar e classificar a perda auditiva" apresentou uma sensibilidade de $35 \%$, especificidade de $25 \%$, um valor preditivo positivo de $12 \%$, valor preditivo negativo de $56 \%$ e coeficiente de concordância Kappa de -0,24. Conclusão: A "ferramenta de detecção de baixo custo para identificar e classificar a perda auditiva" apresenta pouca capacidade para classificar corretamente o doente como verdadeiro doente e ao sadio como verdadeiro sadio. Além disso, a pobre concordância entre os dois exames não permite classificá-la como uma ferramenta válida para a identificação e a classificação da perda auditiva na população infantil colombiana. Considerando estes resultados, recomenda-se que qualquer método de triagem auditiva ou de diagnóstico, deve cumprir com o processo de validação antes de ser aplicado na população. A prevalência de perda auditiva encontrada foi de $23,1 \%$.

-Palavras-chave: Perda auditiva; triagem massiva; audiometria; validade de testes; reprodutibilidade de resultados

\section{Introducción}

El defecto de audición es conocido como "la incapacidad de oír tan bien como una persona cuyo sentido del oído es normal" [1]. Además, "La pérdida de audición puede ser leve, moderada, grave o profunda. Afecta a uno o ambos oídos" [2]. Así mismo, la hipoacusia es considerada una "alteración sensorial muy frecuente del ser humano" y "con numerosas implicaciones médicas, sociales y culturales" [3]. Se estima que, a nivel mundial, de 2 a 3 niños por cada 1000 nacidos vivos tienen algún grado de hipoacusia y "se puede desarrollar en niños que tenían audición normal cuando eran bebes" [4].
En países como Estados Unidos, los reportes alcanzan los 35 millones de norteamericanos con pérdida auditiva; en China, 20 millones; en Europa, 71 millones, y en Latinoamérica, específicamente en Chile, cerca de 1,5 millones de personas presentan este evento [5].

Según los registros del "Análisis de situación de la salud auditiva y comunicativa en Colombia", la prevalencia poblacional para la hipoacusia conductiva es de 109,6 por 100000 habitantes; para la hipoacusia neurosensorial, de 379,8 por 100000 habitantes, y para la hipoacusia mixta, de 135,4 por 100000 habitantes [6]. A nivel local, en Popayán, departamento de Cauca, los reportes son escasos; sin embargo, "según información 
suministrada por la Unidad de Atención Integral (UAI, 2010) se reportaron 7 estudiantes de básica primaria con sordera profunda, distribuidos desde el grado segundo al cuarto correspondientes a diferentes instituciones educativas de la ciudad" [7, p. 17].

El diagnóstico temprano de la hipoacusia se hace indispensable para identificar las posibilidades comunicativas de un individuo. En el caso de un niño es aún más importante, ya que una pérdida auditiva ocasiona retraso en el aprendizaje, deserción escolar y trae consecuencias sociales, emocionales y económicas. En ese sentido, se calcula que "los casos desatendidos de pérdida de audición representan un coste mundial anual de 750000 millones de dólares internacionales" [2].

Para el diagnóstico de la hipoacusia existen varias pruebas formales, como audiometría tonal, otoemisiones acústicas y potenciales auditivos evocados, entre otros, y pruebas informales, como pruebas conductuales y tamizajes [8]. Las pruebas informales por lo general no necesitan de recurso humano profesional, de equipos sofisticados y costosos, y en muchas ocasiones son la única herramienta con que cuenta el personal de atención primaria en salud en las zonas más apartadas.

En Brasil, un grupo de investigadores desarrolló una investigación denominada "Desarrollo y análisis de una herramienta de detección de bajo costo para identificar y clasificar la pérdida auditiva". Esta herramienta, que consta de 15 preguntas, es de fácil acceso, es de bajo costo, su aplicación es sencilla y no necesita de recursos tecnológicos. Además, los investigadores la recomiendan para ser aplicada en los países en desarrollo, como Colombia [9]. Al revisar la literatura, los investigadores de Brasil describen la validación de la herramienta. Sin embargo, no se encontraron estudios que muestren el proceso de validación para Colombia, por lo que se hizo necesario realizar una investigación para establecer la validez de criterio de la herramienta para la detección de la pérdida auditiva en población infantil colombiana.

\section{Metodología}

Se llevó a cabo un estudio de concordancia en una población universo conformada por 692 niños, con edades entre 5 y 10 años, y escolarizados en dos instituciones educativas de la comuna 6 , de estrato socioeconómico 2, de la ciudad de Popayán - Colombia (Antonio García Paredes y Normal Superior).

Para determinar el tamaño de la muestra se utilizó el programa de análisis de datos epidemiológicos de datos tabulados (Epidat 3.1), estableciendo un $95 \%$ de confianza, un poder del $80 \%$ y un $5 \%$ máximo de error.

La muestra estuvo compuesta por 160 niños seleccionados a conveniencia y que cumplieran los siguientes criterios de inclusión: edades comprendidas entre 5 y 10 años, no padecer enfermedades que dificultaran la evaluación y que cumplieran con el consentimiento y el asentimiento firmado por los padres o tutores.

El diagnóstico de la pérdida auditiva de los sujetos de investigación se hizo mediante dos métodos: el primero consistió en el diligenciamiento de las 15 preguntas de la herramienta desarrollada en Brasil, siguiendo estrictamente las instrucciones y los puntos de corte definidos por sus creadores [9] y previamente adaptada culturalmente para la población colombiana. Este proceso fue desarrollado por un grupo de estudiantes de último semestre de la carrera de Fonoaudiología, quienes solicitaron a los padres de los niños seleccionados responder el cuestionario. Los niños cuyo cuestionario arrojaron un puntaje total igual o superior a 6 , se consideraron con pérdida auditiva o hipoacusia.

El segundo método consistió en realizar la audiometría tonal como prueba de oro [10] a cada uno de los niños, utilizando un audiómetro Interacoustics ${ }^{\circledR}$ AD629 y calibrado según el estándar internacional Norma ISO 389. Para este proceso se utilizó la técnica americana, tomando como enfermos con hipoacusia a los niños con umbrales auditivos mayores que $15 \mathrm{~dB}$. Los resultados producto de los dos métodos de diagnóstico fueron revisados por audiólogas expertas, mediante el método de doble ciego, ya que las evaluadoras no conocieron los cuestionarios ni las audiometrías. Los datos fueron tabulados en Excel ${ }^{\circledR}$ 2010, para luego ser analizados.

Para establecer la validez de criterio de la herramienta, se determinó su sensibilidad, especificidad y valores predictivos positivo y negativo. Además, se estimó el grado de concordancia entre los dos métodos diagnósticos, mediante el índice de kappa.

Para el desarrollo de la investigación se tuvieron en cuenta los principios éticos establecidos en la Declaración de Helsinki [11] y la reglamentación de ética colombiana definidos en la Resolución 8430 de 1993 [12]. Además, se obtuvieron el consentimiento y el asentimiento firmado por los padres o tutores. Igualmente, el trabajo de campo solo se llevó a cabo una vez obtenida la autorización por el Comité de Ética de la Universidad del Cauca, mediante Acta 6-1.38 del 17 de diciembre de 2014.

\section{Resultados}

En la Tabla 1 se muestran las características sociodemográficas de los sujetos de investigación. El promedio de edad fue de 7,73 años, con una desviación estándar de $\pm 1,59$; el 54,4\% tenían edades entre 8 y 10 años, y la mayoría (54,4 \%) fueron mujeres. El 61,3\% cursaban los grados de transición a tercero, mientras que el 38,8 $\%$, de cuarto a sexto. Por otra parte, en los 160 niños seleccionados a conveniencia se encontró una prevalencia de pérdida auditiva del $23,1 \%$. 
En la Tabla 2 se presentan los valores de sensibilidad, especificidad y valores predictivos de la herramienta a validar; además, el grado de concordancia entre los dos métodos aplicados. Se encontró que la capacidad de la herramienta para identificar los verdaderos positivos (sujetos que presentan hipoacusia) fue del $35,1 \%$, con un IC $95 \%$ de 18,4 a 51,8 .

Por otra parte, la capacidad de la prueba para identificar los verdaderos negativos (sujetos sanos) fue del $25,2 \%$, con un IC $95 \%$ de 17,1 a 33,2 (véase Tabla 3).

Tabla 1. Características sociodemográficas de la población objeto de estudio.

\begin{tabular}{|c|c|c|c|}
\hline \multicolumn{2}{|c|}{ Variables } & $n=160$ & $\begin{array}{c}\text { Porcentaje } \\
(\%)\end{array}$ \\
\hline \multirow{3}{*}{ Edad en años } & Media $\pm \mathrm{DS}$ & $7,73 \pm$ DS 1,59 & \\
\hline & $5-7$ & 74 & 45,6 \\
\hline & $8-10$ & 87 & 54,4 \\
\hline \multirow{2}{*}{ Sexo } & Hombres & 73 & 45,6 \\
\hline & Mujeres & 87 & 54,4 \\
\hline \multirow{2}{*}{ Grado escolar } & Transición-Tercero & 98 & 61,3 \\
\hline & Cuarto-Sexto & 62 & 38,8 \\
\hline
\end{tabular}

DS: Desviación estándar.

Tabla 2. Sensibilidad, especificidad, valores predictivos y grado de concordancia de la herramienta a validar.

\begin{tabular}{|c|c|c|c|c|c|c|c|c|}
\hline & & \multicolumn{2}{|c|}{$\begin{array}{c}\text { Prueba de oro } \\
\text { (Audiometría tonal) }\end{array}$} & \multirow{2}{*}{$S$} & \multirow{2}{*}{$\mathrm{E}$} & \multirow{2}{*}{$V p+$} & \multirow{2}{*}{$\mathrm{Vp}-$} & \multirow{2}{*}{$\mathrm{K}$} \\
\hline & & Con hipoacusia & $\begin{array}{c}\text { Sin } \\
\text { hipoacusia }\end{array}$ & & & & & \\
\hline \multirow{2}{*}{$\begin{array}{l}\text { Prueba a validar } \\
\text { (Herramienta) }\end{array}$} & Con hipoacusia & 13 & 92 & \multirow{2}{*}{0,35} & \multirow{2}{*}{0,25} & \multirow{2}{*}{0,12} & \multirow{2}{*}{0,56} & \multirow{2}{*}{$-0,24$} \\
\hline & Sin hipoacusia & 24 & 31 & & & & & \\
\hline
\end{tabular}

$\mathrm{S}=$ Sensibilidad, $\mathrm{E}=$ Especificidad, $\mathrm{Vp}+=$ Valor predictivo positivo, $\mathrm{Vp}-=$ Valor predictivo negativo, $\mathrm{K}=$ Índice de kappa .

Tabla 3. Intervalos de confianza de los valores de $\mathrm{S}, \mathrm{E}$, $\mathrm{Vp}+, \mathrm{Vp}-\mathrm{y} \mathrm{K}$ de la herramienta a validar.

\begin{tabular}{lcc}
\hline & Valores & Ic 95 \% \\
\hline Sensibilidad & 35,1 & $(18,4-51,8)$ \\
Especificidad & 25,2 & $(17,1-33,2)$ \\
Índice de validez & 27,5 & $(20,2-34.7)$ \\
Valor predictivo positivo & 12,3 & $(5,6-19,1)$ \\
Valor predictivo negativo & 56,3 & $(92,3-97,3)$ \\
Prevalencia & 23,1 & $(16,2-29,9)$ \\
Razón de verosimilitud positivo & 0,9 & $(0,30-0,79)$ \\
Razón de verosimilitud negativo & 2,5 & $(1,75-3,79)$ \\
\hline
\end{tabular}

En cuanto a los valores predictivos, los resultados arrojaron que existe un $12,3 \%$ de probabilidad de que los sujetos detectados como hipoacúsicos mediante la herramienta, sean realmente hipoacúsicos $(\mathrm{Vp}+)$. Así mismo, hay una probabilidad del $56,3 \%$ de que los sujetos catalogados como sanos mediante la herramienta realmente no presenten hipoacusia $(\mathrm{Vp}-)$.

Con respecto al grado de concordancia, el estudio encontró un índice de kappa menor que $0(K=-0,24)$.
Se encontró, además, una razón de verosimilitud positivo de 0,9 y una razón de verosimilitud negativo de 2,5 , con un IC $95 \%$ de 0,30 a 0,79 , y 1,75 a 3,79 , respectivamente.

\section{Discusión}

Las características sociodemográficas descritas en la población estudiada se asemejan a otros estudios nacionales que investigaron niños entre 5 y 10 años [13-14], y con respecto a la prevalencia de hipoacusia encontrada $(23,1 \%)$, los resultados no difieren de lo reportado a nivel internacional $[5,15,16]$, nacional [6] y local [7,14]. Sin embargo, es importante anotar que la prevalencia hallada mediante la audiometría tonal podría aumentar si se considera realizar audiometrías de alta frecuencias (10 a $16 \mathrm{kHz}$ ), como se ha demostrado en otros estudios [17].

La detección temprana de la hipoacusia siempre ha sido importante para los países y ha sido imperativa sobre todo en la población infantil. Las políticas internacionales [18] y nacionales [19] así lo demuestran. En ese sentido, la investigación permitió establecer que la 
herramienta desarrollada por los investigadores brasileños presentó una sensibilidad y una especificidad menor que el $70 \%$, lo que se traduce en una prueba que no tiene la capacidad para detectar a un enfermo como verdadero enfermo y a un sano como verdadero sano [20]. Estos resultados no concuerdan con la sensibilidad y especificidad reportada para la audiometría tonal ( $\mathrm{S}=96 \%$, $\mathrm{E}=92 \%$ ) [21], siendo considerada como una "prueba esencial en la estimación de la capacidad auditiva [...]" [22, p.10]. Además, utilizar un instrumento con baja especificidad para detectar tempranamente la hipoacusia infantil, "causaría innecesaria preocupación a los padres y recargaría el sistema de salud [...]" [23].

Otros aspectos hallados en esta investigación fueron los valores predictivos positivo y negativo menores que el $57 \%$, lo que indica una probabilidad baja de que los niños reportados como enfermos tengan en realidad hipoacusia y de que los niños reportados como sanos no presenten el evento. Por otro lado, el índice de kappa (< 0 ) demuestra la pobre concordancia entre las dos pruebas [24]. Estos resultados coinciden con la baja sensibilidad, especificidad y valores predictivos encontrados en una investigación de validación de criterio de la "Escala abreviada del desarrollo" (EAD-1), en el dominio de audición y lenguaje, aplicada a niños colombianos menores de 10 años [14]. Lo anterior indica que la valoración de la audición es compleja y, por tanto, la detección temprana de la hipoacusia no se puede realizar mediante escalas con baja sensibilidad y baja especificidad.

En ese sentido, algunos investigadores y especialistas de la audiología sostienen que "la evaluación de la audición es un proceso de alta complejidad y requiere de experticia, precisión, efectividad y veracidad" [22, p. 10]. Además, que se necesita de información clínica de antecedentes y "una variedad de pruebas a nivel comprensivo, las que utilizan estímulos de tonos puros o habla y algunas con uso de ruido enmascarante o de competencia" $[22$, p. 10]. Lo anterior ratifica la importancia de reconocer que las pruebas de tamizaje auditivo utilizadas en la atención primaria en salud deben poseer una alta sensibilidad y especificidad, y no solo ser de bajo costo.

Al respecto, algunos investigadores describen que "el uso de preguntas simples y de encuestas posee la ventaja de su bajo costo y fácil aplicabilidad, pero tienen baja sensibilidad" [21, p. 218]. Entre ellas podemos mencionar la Hearing Handicap Inventory for the Elderly (HHIE) [25] y la Hearing Handicap Inventory for Elderly Screening (HHIE-S) [26], con una sensibilidad que no supera el $75 \%$. Igualmente, algunos investigadores reportan que algunos test pueden ser útiles en estudios poblacionales, pero no permitir "diferenciar en forma objetiva casos de hipoacusia leve respecto de una moderada" [21, p. 218].

\section{Conclusiones}

La herramienta desarrollada en Brasil y recomendada para ser aplicada en países de bajos ingresos, como Colombia, presenta poca capacidad para clasificar correctamente al niño enfermo como verdadero enfermo y al niño sano como verdadero sano. Además, la pobre concordancia con la prueba oro no permite clasificarla como un instrumento válido para la identificación y la clasificación de la pérdida auditiva en niños colombianos con edades entre los 5 y 10 años.

Los resultados obtenidos permiten recomendar que, para la atención primaria en salud auditiva de la población infantil colombiana, se utilicen las pruebas de tecnología blanda y tecnología dura, definidas en el programa de Tamizaje Auditivo Organizado [27], dados sus buenos resultados en su proceso de validación [28], así como los lineamientos definidos en las "Rutas de atención integral en salud", definidas por el Ministerio de Salud de Colombia, en el marco de la nueva "Política de atención integral en salud" [29].

Dada la alta prevalencia de pérdida auditiva encontrada en la población infantil (23,1\%), se hace necesario implementar con urgencia las acciones de promoción de la salud auditiva y comunicativa descritas en el "Plan Decenal de Salud Pública 2012-2021 de Colombia" [30], incluyendo al fonoaudiólogo general como un actor principal dentro de los equipos básicos en atención primaria, e implementar las estrategias "Somos todo oídos" y "Amor por el silencio", y las acciones en el entorno hogar, comunitario, laboral y educativo, definidas por el Ministerio de Salud de Colombia [31].

\section{Limitaciones de estudio}

Como posibles limitaciones del estudio, el grupo investigador considera que al tratarse de un estudio local y no multicéntrico, los valores de sensibilidad, especificidad, valores predictivos y concordancia podrían variar al aumentar el tamaño de la muestra.

Por otro lado, en el estudio solo se estableció la prevalencia de la pérdida auditiva, sin determinar el tipo, el grado y localización de dicha pérdida.

\section{Agradecimientos}

Los autores agradecen a la Universidad del Cauca; a las estudiantes de Fonoaudiología, Angee Vanessa Hincapié, Cristi Carolina Benavides, Yomaira Marisol Jurado, Kelly Johanna Londoño, Leidy Paola Martínez y Nicole Vanessa Solarte y a los padres de familia de los niños intervenidos. 


\section{Declaración de fuente de financiación}

Los autores declaran que la única financiación de tipo económico para este proyecto consistió en la asignación de 2 horas de labor académica asignadas a cada uno de los autores por parte del programa de Fonoaudiología, de la Universidad del Cauca.

\section{Declaración de conflicto de interés}

Los autores manifiestan ser independientes y declaran no tener ningún conflicto de interés.

\section{Declaración de responsabilidad}

Se declara que los puntos de vista expresados son responsabilidad de los autores y no de la institución en la que trabajan o de la fuente de financiación.

\section{Declaración de autoría}

Augusto Muñoz Caicedo. Concepción del proyecto original, análisis e interpretación de resultados, redacción del manuscrito, aprobación final de la versión a publicar y acuerdo de responsabilidad de todos los aspectos del trabajo.

María Consuelo Chaves Peñaranda. Contribuciones sustanciales a la concepción del proyecto, análisis e interpretación de resultados, aprobación final de la versión a publicar y acuerdo de responsabilidad de todos los aspectos del trabajo.

Claudia Ximena Campo Cañar. Contribuciones sustanciales a la concepción del proyecto, análisis e interpretación de resultados, aprobación final de la versión a publicar y acuerdo de responsabilidad de todos los aspectos del trabajo.

\section{Referencias}

1. Organización Mundial de la Salud. La sordera y los defectos de audición [internet]. s. f. [citado 2017 oct. 1]. Disponible en: http:// www.who.int/topics/deafness/es/

2. Organización Mundial de la Salud. Sordera y perdida de la audición [Internet]. 2019 mar. 15 [citado 2020 may. 21]. Disponible en: https://www.who.int/es/news-room/fact-sheets/detail/deafness-and-hearing-loss

3. Alzina V. Detección precoz de la hipoacusia en el recién nacido. An Pedriatr (Barc). 2005;63(3):193-8. Dor: https://doi org/10.1157/13078480

4. Cáceres C, Ruiz J, Solanellas Soler J. Sordera o hipoacusia en el niño pequeño. Madrid: Asociación Española de Pediatría; 2012.

5. Hear-it. La pérdida de audición en distintos países. Bélgica; 1999 [citado 2017 oct. 1]. Disponible en: http://www.hear-it.org/es/laperdida-de-audicion-en-distintos-paises
6. Colombia. Ministerio de Salud, OPS, OMS. Análisis de situación de la salud auditiva y comunicativa en Colombia. Convenio 519 de 2015. Bogotá: El Ministerio [citado 2017 oct. 2]; 2016. Disponible en: https://www.minsalud.gov.co/sites/rid/Lists/BibliotecaDigital/RIDE/VS/PP/ENT/asis-salud-auditiva-2016.pdf

7. Chaves M, Barreto A. Estado auditivo de estudiantes de primer grado según docentes y pruebas audiológicas. Revista Areté [internet]. 2012 [citado 2017 oct. 1]; 12(1):17. Disponible en: http:// revistas.iberoamericana.edu.co/index.php/arete/article/view/352.

8. Gómez O. Audiología básica. La rehabilitación de los problemas auditivos y vestibulares. Bogotá: Universidad Nacional de Colombia [internet]; 2006. [citado 2017 oct. 1]. Disponible en http://www.bdigital.unal.edu.co/3532/1/ Audiolog\%C3\%ADaB $\%$ C3\%A1sica-OGG.pdf

9. Samelli A, Rabello C, Vespasiano A. Development and analysis of a low-cost screening tool to identify and classify hearing loss in children: A proposal for developing countries. Clinical Science. 2011;66(11):1943-9. Disponible en: https://www.ncbi.nlm.nih. gov/pmc/articles/PMC3203968/

10. Pedraza Z, Delgado M. El deficit de audición en la tercera edad. Rev. Fac Med unam [internet]. 2008 [citado 2020 may. 21]; 51(3):91-95. Disponible en: https://www.medigraphic.com/pdfs/ facmed/un-2008/un083b.pdf

11. Asociación Médica Mundial. Declaración de Helsinki. [internet]. s. f. [citado 2020 may.21]. Disponible en: https://www.wma.net/ es/que-hacemos/etica-medica/declaracion-de-helsinki/

12. Colombia. Ministerio de Salud. Resolución 8430, por la cual se establecen las normas científicas, técnicas y administrativas para la investigación en salud. Bogotá [internet]. 1993 (oct. 4) [citado 2020 may. 21]. Disponible en: https://www.minsalud.gov.co/ sites/rid/Lists/BibliotecaDigital/RIDE/DE/DIJ/RESOLUCION8430-DE-1993.pdf

13. Méndez M, Gutiérrez I, Arch E. Manifestaciones conductuales por las cuales se sospecha de hipoacusia en niños. Anales Médicos [internet]. 2003 [citado 2017 oct. 2];48(4):199-203. Disponible en: http://www.medigraphic.com/pdfs/abc/bc-2003/bc034b.pdf

14. Muñoz A, Zapata H, Pérez L. Validación de criterio de la Escala Abreviada del Desarrollo (EAD-1) en el dominio audiciónlenguaje. Rev. Salud Pública [internet]. 2013 [citado 2017 oct. 1]; 15(3):386-97. Disponible en: https://www.scielosp. org/scielo.php?pid=S0124-00642013000300006\&script=sci_ abstract\&tlng=es

15. Asociación Americana del Habla, Lenguaje y Audición (American Speech-Language-Hearing Associtarion, ASHA). Tipo, grado y configuración de la pérdida de audición. Audiología. Serie Informativa [internet]. 2016 [citado 2017 oct. 1]. Disponible en:https:// www.asha.org/uploadedFiles/Tipo-grado-y-configuracion-de-laperdida-de-audicion.pdf

16. Ferrite S, Santana V, Marshall S. Validity of self-reported hearing loss in adults: Performance of three single questions. Rev. Saúde Pública. 2001;45(5):824-30. DoI: https://doi.org/10.1590/S003489102011005000050

17. Rodríguez A. Determinación de los umbrales de audición en la población española. Patrones de normalidad de la totalidad del espectro auditivo humano [Tesis doctoral]. Hospital Universitario Puerta de Hierro. Madrid [internet]. 2015 [citado 2020 feb. 25]. Disponible en: https://repositorio.uam.es/bitstream/hand1e/10486/667533/rodriguez_valiente_antonio.pdf?sequence $=1$

18. Organización Mundial de la Salud. Carta de Ottawa para la promoción de la salud [internet]. 1986 [citado 2020 may. 22]. Disponible en: https://www.paho.org/hq/dmdocuments/2013/Carta-deottawa-para-la-apromocion-de-la-salud-1986-SP.pdf 
19. Saludcolombia.com. Norma técnica para la detección temprana de las alteraciones del crecimiento y desarrollo en el menor de 10 años. [internet]. 2000 [citado 2020 may. 22]. Disponible en: http:// www.saludcolombia.com/actual/htmlnormas/ntcreyde.htm

20. Bravo-Grau S, Cruz JP. Estudios de exactitud diagnóstica: herramientas para su interpretación. Revista Chilena de Radiología [internet]. 2015 [citado 2017 oct. 2]; 21(4):158-64. Disponible en: http://www.scielo.cl/pdf/rchradiol/v21n4/art07.pdf

21. Daszenies C, Lizana M, Cofre N. Validación de la audiometría de vía aérea (AVA) como instrumento de evaluación de hipoacusia en el adulto en Atención Primaria de Salud. Rev. Otorrinolaringol. Cir. Cabeza Cuello [internet]. 2005 [citado 2020 may. 22]; 65:215220. Disponible en: https://www.sochiorl.cl/uploads/09(21).pdf

22. Matos M, Rubiano M. Comparación de resultados de audiometría tonal y test de ruido para igualar umbrales (TEN) en adultos con deficiencia auditiva moderada a severa. Bogotá: Corporación Universitaria Iberoamericana [internet]; 2017 [citado 2020 mar. 1]. Disponible en: https://repositorio.ibero.edu.co/ bitstream/001/781/1/Comparaci\%C3\%B3n\%20de\%20resultados\%20de $\% 20$ audiometr\%C3\%ADa $\% 20$ tonal $\% 20 y \% 20$ test $\% 20$ de $\% 20$ ruido $\% 20$ para $\% 20$ igualar $\% 20$ umbrales $\% 20$ $\% 28$ TEN $\% 29 \% 20$ en $\% 20$ adultos $\% 20$ con $\% 20$ deficiencia $\% 20$ auditiva $\% 20 \mathrm{de} \% 20$ moderada $\% 20 \mathrm{a} \% 20$ severa.pdf

23. Pascucci MC, Lejarraga H, Kelmansky D, et al. Validación de la prueba nacional de pesquisa de trastornos de desarrollo psicomotor en niños menores de 6 años. Rev. Chil. Pediatr [internet]. 2004 [citado 2020 may. 21]; 75(1):75-76. Disponible en: https:// scielo.conicyt.cl/scielo.php?script $=$ sci_arttext\&pid $=\mathrm{S} 0370$ 41062004000100012\&lng=es. http://dx.doi.org/10.4067/S037041062004000100012

24. Landis J, Koch G. The measurement of observer agreement for categorical data. Biometrics. 1977;33(1):159-74. DoI: https://doi. org/10.2307/2529310
25. Ventry IM, Weinstein BE. The hearing handicap inventory for the elderly: A new tool. Ear Hear. 1982;3(43):128-34. Dor: https://doi. org/10.1097/00003446-198205000-00006

26. Gates GA, Murphy M, et al. Screening for handicapping hearing loss in elderly. J Fam Pract. [internet] 2003 [citado 2020 may. 27]; 52(1):56-62. Disponible en: https://pubmed.ncbi.nlm.nih. gov/12540314/

27. Colombia. Ministerio de Salud y Protección Social. Salud auditiva y comunicativa en Colombia. [internet] s. f. [citado 2020. may. 22]. Disponible en: https://www.minsalud.gov.co/sites/rid/Lists/ BibliotecaDigital/RIDE/VS/PP/ENT/alistamiento-operacion-riasac.pdf

28. Colombia. Ministerio de Salud y Protección Social. Dirección de Promoción y Prevención, Subdirección de Enfermedades no Transmisibles. Instrumento de valoración de la audición y lenguaje para niños de 0 a 12 años. Bogotá [internet]; 2016 [citado 2020 may. 22]. Disponible en: https://www.minsalud.gov.co/sites/rid/Lists/BibliotecaDigital/RIDE/VS/PP/ENT/manual-diseno-vale.pdf

29. Colombia. Ministerio de Salud y Protección Social. Políticas de atención integral en salud "Un sistema de salud al servicio de la gente”. Bogotá [internet]; 2016 [citado 2017 oct. 2]. Disponible en: https://www.minsalud.gov.co/sites/rid/Lists/BibliotecaDigital/RIDE/DE/modelo-pais-2016.pdf

30. Colombia. Ministerio de Salud y Protección Social. Plan Decenal de Salud Pública 2012-2021. Bogotá [internet]; 2013 [citado 2020 may. 27]. Disponible en: https://www.minsalud.gov.co/Documentos $\% 20 \mathrm{y} \% 20$ Publicaciones/Plan $\% 20$ Decenal $\% 20$ - $\% 20$ Documento $\% 20$ en $\% 20$ consulta $\% 20$ para $\% 20$ aprobaci $\% \mathrm{C} 3 \%$ B3n.pdf

31. Colombia. Ministerio de Salud. Lineamientos para la promoción y gestión integral de la salud auditiva y comunicativa. "Somos todos oídos”. Bogotá [internet]; 2014 [citado 2017 oct. 1]. Disponible en: https://www.minsalud.gov.co/sites/rid/Lists/BibliotecaDigital/RIDE/VS/PP/ENT/lineamientos-salud-auditiva-guiametodologica-2017.pdf 